PROCEEDINGS OF THE

AMERICAN MATHEMATICAL SOCIETY

Volume 138, Number 2, February 2010, Pages 385-393

S 0002-9939(09)10144-2

Article electronically published on October 2, 2009

\title{
THE $q$-TANGENT AND $q$-SECANT NUMBERS VIA BASIC EULERIAN POLYNOMIALS
}

\author{
DOMINIQUE FOATA AND GUO-NIU HAN
}

(Communicated by Jim Haglund)

\begin{abstract}
The classical identity that relates Eulerian polynomials to tangent numbers together with the parallel result dealing with secant numbers is given a $q$-extension, both analytically and combinatorially. The analytic proof is based on a recent result by Shareshian and Wachs and the combinatorial one on the geometry of alternating permutations.
\end{abstract}

\section{INTRODUCTION}

Let $\left(A_{n}(s, Y)\right)(n \geq 0)$ be the sequence of polynomials in two variables defined by

$$
\sum_{n \geq 0} \frac{u^{n}}{n !} A_{n}(s, Y)=\frac{1-s}{\exp (s u)-s \exp (u)} \exp (Y u)
$$

It is known (see, e.g., 11], chap. 4) that they are polynomials with positive integral coefficients. For $Y=1$ we recover the Eulerian polynomials $\left(A_{n}(s, 1)\right)$ introduced by Euler $\left[8\right.$ for his evaluation of the alternating sum $\sum_{i=1}^{m}(-1)^{i} i^{n}$. Moreover, Euler derived the two identities

$$
A_{2 n}(-1,1)=0 \quad(n \geq 1) ; \quad(-1)^{n} A_{2 n+1}(-1,1)=T_{2 n+1} \quad(n \geq 0),
$$

where $T_{2 n+1}(n \geq 0)$ are the coefficients of the Taylor expansion of $\tan u$,

$$
\begin{aligned}
\tan u & =\sum_{n \geq 0} \frac{u^{2 n+1}}{(2 n+1) !} T_{2 n+1} \\
& =\frac{u}{1 !} 1+\frac{u^{3}}{3 !} 2+\frac{u^{5}}{5 !} 16+\frac{u^{7}}{7 !} 272+\frac{u^{9}}{9 !} 7936+\frac{u^{11}}{11 !} 353792+\cdots .
\end{aligned}
$$

The coefficients $T_{2 n+1}(n \geq 0)$, called tangent numbers, are positive integral numbers, and so are the secant numbers $E_{2 n}(n \geq 1)$ (see, e.g., [16, p. 177) defined by

Received by the editors October 6, 2008.

2000 Mathematics Subject Classification. Primary 05A15, 05A30, 05E15.

Key words and phrases. $q$-tangent numbers, $q$-secant numbers, $q$-Eulerian polynomials, excedances, derangements, desarrangements, alternating permutations.

(C)2009 American Mathematical Society 
the Taylor expansion of $\sec u$ :

$$
\begin{aligned}
\sec u=\frac{1}{\cos u} & =1+\sum_{n \geq 1} \frac{u^{2 n}}{(2 n) !} E_{2 n} \\
& =1+\frac{u^{2}}{2 !} 1+\frac{u^{4}}{4 !} 5+\frac{u^{6}}{6 !} 61+\frac{u^{8}}{8 !} 1385+\frac{u^{10}}{10 !} 50521+\cdots .
\end{aligned}
$$

The parallel result to (1.2) involving secant numbers was obtained by Roselle [18, for the polynomials $A_{n}(s, 0)$ in the form

$$
A_{2 n-1}(-1,0)=0 \quad(n \geq 1) ; \quad(-1)^{n} A_{2 n}(-1,0)=E_{2 n} \quad(n \geq 0) .
$$

For each permutation $\sigma=\sigma(1) \sigma(2) \cdots \sigma(n)$ of $12 \cdots n$, the number of excedances $\operatorname{exc} \sigma$ (resp. of descents, $\operatorname{des} \sigma$ ) of $\sigma$ is defined to be the number of integers $i$ such that $1 \leq i \leq n-1$ and $\sigma(i)>i$ (resp. $\sigma(i)>\sigma(i+1)$ ). Also, fix $\sigma$ designates the number of fixed points of $\sigma$ and $\mathfrak{D}_{n}$ the subset of $\mathfrak{S}_{n}$ of the permutations without fixed points, called derangements. It is known that the two statistics "exc" and "des" have the same distributions over the symmetric group (15, p. 186). As derived in [11, we have $A_{n}(s, Y)=\sum_{\sigma \in \mathfrak{S}_{n}} s^{\operatorname{exc} \sigma} Y^{\text {fix } \sigma}$. The specializations are due to Riordan (17], p. 214) for $Y=1$ and to Roselle [18] for $Y=0$.

The combinatorial counterparts of (1.2) and (1.5) can be expressed as

$$
\begin{aligned}
\sum_{\sigma \in \mathfrak{S}_{2 n}}(-1)^{\operatorname{exc} \sigma} & =0(n \geq 1) ; \\
\sum_{\sigma \in \mathfrak{S}_{2 n+1}}(-1)^{n+\operatorname{exc} \sigma} & =T_{2 n+1}(n \geq 0) ; \\
\sum_{\sigma \in \mathfrak{D}_{2 n-1}}(-1)^{\operatorname{exc} \sigma} & =0(n \geq 1) ; \\
\sum_{\sigma \in \mathfrak{D}_{2 n}}(-1)^{n+\operatorname{exc} \sigma} & =E_{2 n}(n \geq 0) .
\end{aligned}
$$

Let $(t ; q)_{n}=(1-t)(1-t q) \cdots\left(1-t q^{n-1}\right)$ for $n \geq 1$ and $(t ; q)_{0}=1$ denote the traditional $q$-ascending factorials. The purpose of this paper is to construct a $q$ analog of (1.6)-(1.9), as further stated in Theorem 1. This first means that two sequences of polynomials $\left(T_{2 n+1}(q)\right),\left(E_{2 n}(q)\right)$ with positive integral coefficients are to be introduced such that $T_{2 n+1}(1)=T_{2 n+1}$ and $E_{2 n}(1)=E_{2 n}$. This is achieved by taking the $q$-tangent numbers $T_{2 n+1}(q)$ and the $q$-secant numbers $E_{2 n}(q)$ (see [3], [4, 9]) occurring in the two expansions:

$$
\begin{gathered}
\tan _{q}(u)=\frac{\sum_{n \geq 0}(-1)^{n} u^{2 n+1} /(q ; q)_{2 n+1}}{\sum_{n \geq 0}(-1)^{n} u^{2 n} /(q ; q)_{2 n}}=\sum_{n \geq 0} \frac{u^{2 n+1}}{(q ; q)_{2 n+1}} T_{2 n+1}(q) ; \\
\sec _{q}(u)=\frac{1}{\sum_{n \geq 0}(-1)^{n} u^{2 n} /(q ; q)_{2 n}}=\sum_{n \geq 0} \frac{u^{2 n}}{(q ; q)_{2 n}} E_{2 n}(q) .
\end{gathered}
$$

Note that $\tan _{q}(u)\left(\right.$ resp. $\left.\sec _{q}(u)\right)$ is simply the quotient of the $q$-sine and $q$-cosine (resp. the inverse of $q$-cosine), as introduced by Jackson [14] (also see [12, p. 23). The first values are: $T_{1}(q)=1 ; T_{3}(q)=q+q^{2} ; T_{5}(q)=q^{2}+2 q^{3}+3 q^{4}+4 q^{5}+3 q^{6}+$ $2 q^{7}+q^{8} ; E_{0}(q)=E_{2}(q)=1, E_{4}(q)=q(1+q)^{2}+q^{4} ; E_{6}(q)=q^{2}(1+q)^{2}\left(1+q^{2}+\right.$ $\left.q^{4}\right)\left(1+q+q^{2}+2 q^{3}\right)+q^{12}$. 
Second, another statistic on the symmetric group $\mathfrak{S}_{n}$ is to be associated with "exc," so that the left-hand sides of the four identities (1.6)-(1.9) will become true polynomial identities. The statistic that meets our expectations is the classical major index "maj," defined for each permutation $\sigma=\sigma(1) \sigma(2) \cdots \sigma(n)$ as the sum of all the $i$ 's such that $\sigma(i)>\sigma(i+1)$. The main result of the present paper is then the following theorem.

Theorem 1. We have:

$$
\begin{aligned}
\sum_{\sigma \in \mathfrak{S}_{2 n}}(-1)^{\operatorname{exc} \sigma} q^{(\mathrm{maj}-\mathrm{exc}) \sigma} & =0 \quad(n \geq 1) ; \\
\sum_{\sigma \in \mathfrak{S}_{2 n+1}}(-1)^{n+\operatorname{exc} \sigma} q^{(\mathrm{maj}-\mathrm{exc}) \sigma} & =T_{2 n+1}(q) \quad(n \geq 0) ; \\
\sum_{\sigma \in \mathfrak{D}_{2 n-1}}(-1)^{\operatorname{exc} \sigma} q^{(\mathrm{maj}-\mathrm{exc}) \sigma} & =0 \quad(n \geq 1) ; \\
\sum_{\sigma \in \mathfrak{D}_{2 n}}(-1)^{n+\operatorname{exc} \sigma} q^{(\mathrm{maj}-\mathrm{exc}) \sigma} & =E_{2 n}(q) \quad(n \geq 0) .
\end{aligned}
$$

We provide two proofs of Theorem 1. The analytic proof, given in Section 2, is based on a recent result due to Shareshian and Wachs [19 who made an explicit study of the statistic "maj-exc". The combinatorial proof, given in Section 4, is based on the geometry of alternating permutations, as introduced by André 1], 2 and on an equidistribution property between two three-variable statistics (exc, fix, maj) and (lec, pix, inv) established in 10. However, to make this paper self-contained, we give a new proof of that equidistribution property by directly calculating the distribution of the latter statistic (see Theorem 4, Section 3). Our combinatorial proof consists of reducing the four alternating sums (1.10)-(1.13) by means of two explicit sign-reversing involutions. In contrast to the combinatorial proofs of (1.6) and (1.7) given in [11], chap. 5, these involutions naturally lead to the alternating permutation model.

\section{The AnAlytic PRoof}

The $q$-analogs of the Eulerian polynomials, which have been derived, are the generating polynomials for $\mathfrak{S}_{n}$ by the pair (des, maj) (Carlitz [5], 6]), by (des, inv) (Stanley [20]) and by (exc, maj) (Shareshian and Wachs [19]). Let us recall the latest $q$-extension.

Theorem 2 (Shareshian-Wachs). Let

$$
A_{n}(s, Y, q)=\sum_{\sigma \in \mathfrak{S}_{n}} s^{\operatorname{exc} \sigma} Y^{\mathrm{fix} \sigma} q^{\operatorname{maj} \sigma} .
$$

Then

$$
\sum_{n \geq 0} \frac{u^{n}}{(q ; q)_{n}} A_{n}(s, Y, q)=\frac{1-s q}{e_{q}(s q u)-s q e_{q}(u)} e_{q}(Y u),
$$

where $e_{q}(u)=\sum_{n \geq 0} u^{n} /(q ; q)_{n}$ is the first q-exponential.

To prove Theorem 1 it then suffices to establish the following equivalent theorem. 
Theorem 3. Let $\left(A_{n}(s, Y, q)\right)(n \geq 0)$ be the sequence of polynomials defined by (2.1). Then

$$
\begin{gathered}
A_{2 n}\left(-q^{-1}, 1, q\right)=0(n \geq 1) ; \quad(-1)^{n} A_{2 n+1}\left(-q^{-1}, 1, q\right)=T_{2 n+1}(q)(n \geq 0) ; \\
A_{2 n-1}\left(-q^{-1}, 0, q\right)=0(n \geq 1) ; \quad(-1)^{n} A_{2 n}\left(-q^{-1}, 0, q\right)=E_{2 n}(q)(n \geq 0) .
\end{gathered}
$$

Proof. With the substitutions $Y:=0$ and $s:=-q^{-1}$ in (2.2) we get

$$
\sum_{n \geq 0} \frac{u^{n}}{(q ; q)_{n}} A_{n}\left(-q^{-1}, 0, q\right)=\left(1+\sum_{n \geq 1} \frac{u^{2 n}}{(q ; q)_{2 n}}\right)^{-1} .
$$

As the fraction on the right involves only even powers of $u$, we deduce:

$$
A_{2 n-1}\left(-q^{-1}, 0, q\right)=0 \quad(n \geq 1) .
$$

By replacing $u$ by $i u$ we obtain

$$
\sum_{n \geq 0} \frac{u^{2 n}}{(q ; q)_{2 n}}(-1)^{n} A_{2 n}\left(-q^{-1}, 0, q\right)=\left(1+\sum_{n \geq 1}(-1)^{n} \frac{u^{2 n}}{(q ; q)_{2 n}}\right)^{-1}=\frac{1}{\cos _{q}(u)} .
$$

Consequently, $(-1)^{n} A_{2 n}\left(-q^{-1}, 0, q\right)=E_{2 n}(q) \quad(n \geq 0)$.

From (2.2) we can write

$$
\sum_{n \geq 0} \frac{u^{n}}{(q ; q)_{n}} A_{n}(s, 1, q)=e_{q}(u) \sum_{n \geq 0} \frac{u^{n}}{(q ; q)_{n}} A_{n}(s, 0, q),
$$

so that by replacing $s$ by $-q^{-1}$ and $u$ by $i u$,

$$
\sum_{n \geq 0} \frac{(i u)^{n}}{(q ; q)_{n}} A_{n}\left(-q^{-1}, 1, q\right)=\frac{e_{q}(i u)}{\cos _{q}(u)}=\frac{\cos _{q}(u)+i \sin _{q}(u)}{\cos _{q}(u)} .
$$

By selecting the real and imaginary parts we get

$$
\sum_{n \text { even }} \frac{(i u)^{n}}{(q ; q)_{n}} A_{n}\left(-q^{-1}, 1, q\right)=1
$$

and

$$
\sum_{n \geq 0} \frac{u^{2 n+1}}{(q ; q)_{2 n+1}}(-1)^{n} A_{2 n+1}\left(-q^{-1}, 1, q\right)=\frac{\sin _{q}(u)}{\cos _{q}(u)}=\tan _{q}(u) .
$$

We then conclude

$$
\begin{aligned}
A_{2 n}\left(-q^{-1}, 1, q\right) & =0 \quad(n \geq 1), \\
(-1)^{n} A_{2 n+1}\left(-q^{-1}, 1, q\right) & =T_{2 n+1}(q) \quad(n \geq 0) .
\end{aligned}
$$

\section{A DiRECT DERIVATION}

A word $w=x_{1} x_{2} \cdots x_{m}$ is called a hook if $x_{1}>x_{2}$ and either $m=2$, or $m \geq 3$ and $x_{2}<x_{3}<\cdots<x_{m}$. As proved by Gessel [13, each permutation $\sigma=\sigma(1) \sigma(2) \cdots \sigma(n)$ admits a unique factorization, called its hook factorization, $p \tau_{1} \tau_{2} \cdots \tau_{k}$, where $p$ is an increasing word and each factor $\tau_{1}, \tau_{2}, \ldots, \tau_{k}$ is a hook. To derive the hook factorization of a permutation, it suffices to start from 
the right and at each step determine the right factor, which is a hook. For each $i$ let inv $\tau_{i}$ denote the number of inversions of $\tau_{i}$ and define

$$
\begin{aligned}
\operatorname{lec} \sigma & :=\sum_{1 \leq i \leq k} \operatorname{inv} \tau_{i} ; \\
\operatorname{pix} \sigma & :=\text { length of the factor } p .
\end{aligned}
$$

Those two statistics have been introduced and used in [10. Furthermore, let Desar $_{n}$ be the subset of $\mathfrak{S}_{n}$ of the permutations $\sigma$, called desarrangements, having the property that $\operatorname{pix} \sigma=07$.

For instance, the hook factorization of the following permutation is indicated by vertical bars: $\sigma=13414|12251115| 867 \mid 13910$. We have $p=13414$, so that $\operatorname{pix} \sigma=4, \operatorname{inv}(12251115)=3, \operatorname{inv}(867)=2, \operatorname{inv}(13910)=2$, so that $\operatorname{lec} \sigma=7$. The next theorem, already derived in [10, is given a new direct proof.

Theorem 4. Let

$$
A_{n}^{\text {lec,pix,inv }}(s, Y, q):=\sum_{\sigma \in \mathfrak{S}_{n}} s^{\operatorname{lec} \sigma} Y^{\text {pix } \sigma} q^{\text {inv } \sigma}
$$

Then

$$
\sum_{n \geq 0} \frac{u^{n}}{(q ; q)_{n}} A_{n}^{\text {lec,pix,inv }}(s, Y, q)=\frac{1-s q}{e_{q}(s q u)-s q e_{q}(u)} e_{q}(Y u)
$$

Proof. Let $p \tau_{1} \tau_{2} \cdots \tau_{r}$ be the hook factorization of a permutation $\sigma$ from $\mathfrak{S}_{n}$. Let $A_{0}$ (resp. $A_{i}(1 \leq i \leq r)$ ) denote the set of all letters in the word $p$ (resp. in the hook $\tau_{i}$ ) and call content of $\sigma$ the sequence Cont $\sigma=\left(A_{0}, A_{1}, \ldots, A_{r}\right)$. Note that $\# A_{i} \geq 2$ for $i=1, \ldots, r$ and $\left(A_{0}, A_{1}, \ldots, A_{r}\right)$ is an ordered partition of $[n]=\{1,2, \ldots, n\}$. The statistic (inv - lec) $\sigma$ is equal to the number of pairs $(k, l)$ such that $k \in A_{i}$, $l \in A_{j}, k>l$ and $i<j$, a number we shall denote by $\operatorname{inv}\left(A_{0}, A_{1} \ldots, A_{r}\right)$.

If $A_{i}=\left\{a_{1}<a_{2}<\cdots<a_{m}\right\}$, then the hooks with content $A_{i}$ are the $(m-1)$ words: $w_{1}=a_{m} a_{1} \cdots a_{m-2} a_{m-1}, w_{2}=a_{m-1} a_{1} \cdots a_{m-2} a_{m}, \ldots, w_{m-1}=$ $a_{2} a_{1} a_{3} \cdots a_{m-1} a_{m}$, whose "lec" statistic and inversion number are both equal to $(m-1),(m-2), \ldots, 1$, respectively. The generating polynomial for those $(m-1)$ hooks by the pair (lec, inv) is then equal to

$$
P_{m}(s, q):=s q+(s q)^{2}+\cdots+(s q)^{m-1}=\frac{s q-(s q)^{m}}{1-s q} .
$$

The identity $\sum_{\substack{\left.A_{0}, A_{1}, \ldots, A_{r}\right) \\ \# A_{i}=a_{i}}} q^{\operatorname{inv}\left(A_{0}, A_{1}, \ldots, A_{r}\right)}=\left[\begin{array}{c}n \\ a_{0}, a_{1}, \ldots, a_{r}\end{array}\right]$, where the right-hand side is the $q$-multinomial coefficient $(q ; q)_{n} /\left((q ; q)_{a_{0}}(q ; q)_{a_{1}} \cdots(q ; q)_{a_{r}}\right)$ is easy to verify. 
We then have

$$
\begin{aligned}
& A_{n}^{\text {lec,pix,inv }}(s, Y, q)=\sum_{\sigma \in \mathfrak{S}_{n}} q^{(\mathrm{inv}-\mathrm{lec}) \sigma}(s q)^{\operatorname{lec} \sigma} Y^{\mathrm{pix} \sigma} \\
& =\sum_{\left(A_{0}, A_{1}, \ldots, A_{r}\right)} q^{\operatorname{inv}\left(A_{0}, A_{1}, \ldots, A_{r}\right)} Y^{\# A_{0}} \sum_{\text {Cont } \sigma=\left(A_{0}, A_{1}, \ldots, A_{r}\right)}(s q)^{\operatorname{lec} \sigma} \\
& =\sum_{\left(A_{0}, A_{1}, \ldots, A_{r}\right)} q^{\operatorname{inv}\left(A_{0}, A_{1}, \ldots, A_{r}\right)} Y^{\# A_{0}} \prod_{1 \leq i \leq r} P_{\# A_{i}}(s, q) \\
& =\sum_{\substack{a_{0}+a_{1}+\ldots+a_{r}=n \\
a_{i} \geq 2}} Y^{a_{0}} \prod_{1 \leq i \leq r)} P_{a_{i}}(s, q) \sum_{\substack{\left(1 \leq i \leq r \\
\left(A_{0}, A_{1}, \ldots, A_{r}\right) \\
\# A_{i}=a_{i}\right.}} q^{\operatorname{inv}\left(A_{0}, A_{1}, \ldots, A_{r}\right)} \\
& =\sum_{\substack{a_{0}+a_{1}+\cdots+a_{r}=n \\
a_{i} \geq 2}}\left[\begin{array}{c}
n \\
\left.a_{0}, a_{1}, \ldots, a_{r} \leq r\right)
\end{array}\right]_{q} Y^{a_{0}} \prod_{1 \leq i \leq r} P_{a_{i}}(s, q) .
\end{aligned}
$$

The rest of the calculation is routine:

$$
\begin{aligned}
& \sum_{n \geq 0} A_{n}^{\text {lec,pix,inv }}(s, Y, q) \frac{u^{n}}{(q ; q)_{n}}=\sum_{\substack{n \geq 0 \\
a_{0}+a_{1}+\ldots+a_{r}=n \\
a_{i} \geq 2}} \sum_{\substack{a_{0} \\
(1 \leq i \leq r)}} \frac{u^{a_{0}}}{(q ; q)_{a_{0}}} \prod_{1 \leq i \leq r} P_{a_{i}}(s, q) \frac{u^{a_{i}}}{(q ; q)_{a_{i}}} \\
& =\left(\sum_{a_{0} \geq 0} \frac{(Y u)^{a_{0}}}{(q ; q)_{a_{0}}}\right)\left(1-\sum_{b \geq 2} P_{b}(s, q) \frac{u^{b}}{(q ; q)_{b}}\right)^{-1} \\
& =e_{q}(Y u)\left(1-\sum_{b \geq 2} \frac{s q-(s q)^{b}}{1-s q} \frac{u^{b}}{(q ; q)_{b}}\right)^{-1} \\
& =e_{q}(Y u) \frac{1-s q}{e_{q}(s q u)-s q e_{q}(u)} \text {. }
\end{aligned}
$$

In view of $(2.1),(2.2),(3.3)$ and (3.4) the following identity holds:

$$
\sum_{\sigma \in \mathfrak{S}_{n}} s^{\operatorname{exc} \sigma} Y^{\mathrm{fix} \sigma} q^{\operatorname{maj} \sigma}=\sum_{\sigma \in \mathfrak{S}_{n}} s^{\operatorname{lec} \sigma} Y^{\operatorname{pix} \sigma} q^{\text {inv } \sigma},
$$

and Theorem 1 is proved if we establish the following four identities:

$$
\begin{aligned}
\sum_{\sigma \in \mathfrak{S}_{2 n}}(-1)^{\operatorname{lec} \sigma} q^{(\mathrm{inv}-\mathrm{lec}) \sigma} & =0 \quad(n \geq 1) ; \\
\sum_{\sigma \in \mathfrak{S}_{2 n+1}}(-1)^{n+\operatorname{lec} \sigma} q^{(\mathrm{inv}-\mathrm{lec}) \sigma} & =T_{2 n+1}(q) \quad(n \geq 0) ; \\
\sum_{\sigma \in \operatorname{Desar}_{2 n-1}}(-1)^{\operatorname{lec} \sigma} q^{(\mathrm{inv}-\mathrm{lec}) \sigma} & =0 \quad(n \geq 1) ; \\
\sum_{\sigma \in \operatorname{Desar}_{2 n}}(-1)^{n+\operatorname{lec} \sigma} q^{(\mathrm{inv}-\text { lec }) \sigma} & =E_{2 n}(q) \quad(n \geq 0) .
\end{aligned}
$$

This is the object of the next section. 


\section{The COMBInAtorial PROOF}

An alternating (resp. falling alternating) permutation is defined to be a permutation $\sigma=\sigma(1) \cdots \sigma(n)$ having the following properties: $\sigma(1)<\sigma(2), \sigma(2)>$ $\sigma(3), \sigma(3)<\sigma(4)$, etc. (resp. $\sigma(1)>\sigma(2), \sigma(2)<\sigma(3), \sigma(3)>\sigma(4)$, etc.) in an alternating way. The set of alternating (resp. falling alternating) permutations of order $n$ is denoted by $\mathfrak{T}_{n}$ (resp. by $\mathfrak{T}_{n}^{\prime}$ ). The combinatorial interpretations $\# \mathfrak{T}_{2 n+1}=\# \mathfrak{T}_{2 n+1}^{\prime}=T_{2 n+1}, \# \mathfrak{T}_{2 n}=\# \mathfrak{T}_{2 n}^{\prime}=E_{2 n}$ are due to Désiré André [1], [2]. For each permutation $\sigma$ let inv $\sigma$ denote the number of its inversions. The following theorem is of common knowledge today, once we know how to $q$-transpose the calculation made by André in his memoirs (see, e.g., [3], Proposition 4.1).

Theorem 5. For each $n \geq 0$ we have

$$
\sum_{\sigma \in \mathfrak{T}_{2 n+1}} q^{\mathrm{inv} \sigma}=\sum_{\sigma \in \mathfrak{T}^{\prime}{ }_{2 n+1}} q^{\mathrm{inv} \sigma}=T_{2 n+1}(q)
$$

and

$$
\sum_{\sigma \in \mathfrak{T}_{2 n}} q^{\mathrm{inv} \sigma}=E_{2 n}(q)
$$

We first prove identities (3.9) and (3.10). With the notation of the previous section the content of each desarrangement is of the form $\left(\emptyset, A_{1}, \ldots, A_{r}\right)$, so that

$$
\begin{aligned}
& \sum_{\sigma \in \operatorname{Desar}_{n}}(-1)^{\text {lec } \sigma} q^{(\mathrm{inv}-\mathrm{lec}) \sigma}=A_{n}^{\text {lec,pix,inv }}\left(-q^{-1}, 0, q\right) \\
& =\sum_{\substack{a_{1}+\cdots+a_{r}=n \\
a_{i} \geq 2}} \prod_{\substack{1 \leq i \leq r \\
A^{\prime}}} P_{a_{i}}\left(-q^{-1}, q\right) \sum_{\begin{array}{c}
\left(A_{1}, \ldots, A_{r}\right) \\
\# A_{i}=a_{i}
\end{array}} q^{\operatorname{inv}\left(A_{1}, \ldots, A_{r}\right)} .
\end{aligned}
$$

From the very definition of $P_{m}(s, q)$ we have

$$
P_{m}\left(-q^{-1}, q\right)=(-1)^{\text {inv } w_{1}}+\cdots+(-1)^{\text {inv } w_{m-1}}= \begin{cases}0, & \text { if } m \text { odd } \\ -1, & \text { if } m \text { even }\end{cases}
$$

Hence, if the ordered partition $\left(A_{1}, A_{2}, \ldots, A_{r}\right)$ has at least one block of odd cardinality and $\# A_{i}=a_{i}(1 \leq i \leq r)$, the product $\prod_{1 \leq i \leq r} P_{a_{i}}\left(-q^{-1}, q\right)$ is null. As each desarrangement from $\mathfrak{S}_{2 n-1}$ has at least one hook of odd length, the sum $\sum_{\sigma \in \operatorname{Desar}_{2 n-1}}(-1)^{\operatorname{lec} \sigma} q^{(\mathrm{inv}-\mathrm{lec}) \sigma}$ is zero. This already proves identity $(3.9)$.

Next, consider the even case. If all the blocks $A_{1}, A_{2}, \ldots, A_{r}$ of the ordered partition $\left(A_{1}, A_{2}, \ldots, A_{r}\right)$ are of even cardinality, then

$$
\sum_{\sigma \in \operatorname{Cont}\left(A_{1}, \ldots, A_{r}\right)}(-1)^{n+\operatorname{lec} \sigma} q^{(\mathrm{inv}-\mathrm{lec}) \sigma}=(-1)^{n+r} q^{\operatorname{inv}\left(A_{1}, \ldots, A_{r}\right)} .
$$

Hence,

$$
\sum_{\sigma \in \operatorname{Desar}_{2 n}}(-1)^{n+\operatorname{lec} \sigma} q^{(\mathrm{inv}-\mathrm{lec}) \sigma}=\sum_{\substack{\left(A_{1}, \ldots, A_{r}\right) \\ \# A_{i} \text { even }}}(-1)^{n+r} q^{\operatorname{inv}\left(A_{1}, \ldots, A_{r}\right)} .
$$

Sign-reversing involution. An ordered partition $\Omega=\left(A_{1}, A_{2}, \ldots, A_{r}\right)$ is said to have an increase at $i$ if $1 \leq i \leq r-1$ and $\max A_{i}<\min A_{i+1}$. If it has no increase and all its blocks $A_{j}$ are of cardinality 2 , then $r=n$ and the corresponding term in (4.1) is equal to $q^{\text {inv } \Omega}$. If it is not the case, let $i$ be the integer with the following properties: 
(i) $\# A_{1}=\cdots=\# A_{i-1}=2$;

(ii) no increase at $1,2, \ldots,(i-1)$;

(iii) either $\# A_{i} \geq 4$ or

(iv) $\# A_{i}=2$ and there is an increase at $i$.

Say that the partition $\Omega$ is of class $C_{i}$ (resp. $C_{i}^{\prime}$ ) if (i), (ii) and (iii) (resp. and (iv)) hold. If $\Omega$ is of class $C_{i}$, let $A_{i}=\left\{a_{1}<a_{2}<\cdots<a_{2 m}\right\}(m \geq 2)$ and form $\Omega^{\prime}=$ $\left(A_{1}, \ldots, A_{i-1},\left\{a_{1}, a_{2}\right\},\left\{a_{3}, \ldots, a_{2 m}\right\}, A_{i+1}, \ldots, A_{r}\right)$. Then, $\Omega^{\prime}$ is of class $C_{i}^{\prime}$. Thus $\Omega \mapsto \Omega^{\prime}$ is an involution of $C_{i}$ onto $C_{i}^{\prime}$, which is sign-reversing since inv $\Omega^{\prime}=\operatorname{inv} \Omega$ and $(-1)^{n+r} q^{\text {inv } \Omega}+(-1)^{n+r+1} q^{\text {inv } \Omega^{\prime}}=0$.

By applying the involution $\Omega \mapsto \Omega^{\prime}$, the only partitions $\left(A_{1}, \ldots, A_{r}\right)$ remaining in (4.1) are of the form $\Omega=\left(\left\{a_{1}<b_{1}\right\},\left\{a_{2}<b_{2}\right\}, \ldots,\left\{a_{n}<b_{n}\right\}\right)$ having the property that for each $i=2, \ldots, n$ the relation $b_{i-1}>a_{i}$ holds. Hence, the permutation $\omega=a_{1} b_{1} a_{2} b_{2} \cdots a_{n} b_{n}$ is an alternating permutation starting with a rise $a_{1}<b_{1}$. We have then proved

$$
\sum_{\sigma \in \operatorname{Desar}_{2 n}}(-1)^{n+\operatorname{lec} \sigma} q^{(\mathrm{inv}-\mathrm{lec}) \sigma}=\sum_{\omega} q^{\mathrm{inv} \omega}
$$

where the sum is over all alternating permutations of order $2 n$, which establishes identity (3.10) by Theorem 5 .

Next, consider the left-hand side (LHS) of (3.8) (resp. of (3.7)). The sum may be regarded as being over all hook factorizations $p \tau_{1} \tau_{2} \cdots \tau_{r}$ of permutations from $\mathfrak{S}_{2 n+1}\left(\operatorname{resp} . \mathfrak{S}_{2 n}\right)$. Let $A_{0}$ be the set of the letters in $p$ and, as before, let $A_{i}$ be the set of all letters in $\tau_{i}(1 \leq i \leq r)$. As above, we may apply the involution $\Omega \mapsto \Omega^{\prime}$ to all contents $\Omega=\left(A_{1}, A_{2}, \ldots, A_{r}\right)$, remembering that this time $\Omega$ is an ordered partition of $[2 n+1] \backslash A_{0}$ (resp. of $[2 n] \backslash A_{0}$ ). After applying $\Omega \mapsto \Omega^{\prime}$ we get

$$
\mathrm{LHS}=\sum_{\left(A_{0}, A_{1}, \ldots, A_{r}\right)}(-1)^{n+r} q^{\operatorname{inv}\left(A_{0}, A_{1}, \ldots, A_{r}\right)}
$$

where the sum is over all ordered partitions $\Theta=\left(A_{0}, A_{1}, \ldots, A_{r}\right)$ of $[2 n+1]$ (resp. of $[2 n]$ with the convention that $A_{0}$ may be empty), having the property that $\left(A_{1}, \ldots, A_{r}\right)$ is an ordered partition of a subset of $[2 n+1]$ (resp. of $\left.[2 n]\right)$ into blocks of cardinality 2 having no increase.

Another sign-reversing involution. Each ordered partition $\Theta=\left(A_{0}, A_{1}, \ldots, A_{r}\right)$ of $[2 n+1]$ (resp. of $[2 n]$ ) is said to be of type $D$, if $\max A_{0}<\min A_{1}$ (by convention, $\max \emptyset=-\infty)$. It is of type $D^{\prime \prime}$ if $\max A_{0}<\min A_{1}$ does not hold and $\# A_{0} \geq 2$. If $\Theta$ is of type $D$, define $\Theta^{\prime \prime}=\left(A_{0} \cup A_{1}, A_{2}, \ldots, A_{r}\right)$. Then, $\Theta^{\prime \prime}$ is of type $D^{\prime \prime}$ and $\operatorname{inv} \Theta^{\prime \prime}=\operatorname{inv} \Theta$, so that

$$
(-1)^{n+r} q^{\operatorname{inv} \Theta}+(-1)^{n+r-1} q^{\text {inv } \Theta^{\prime \prime}}=0 .
$$

Thus $\Theta \mapsto \Theta^{\prime \prime}$ is a sign-reversing involution.

After applying the transformation $\Theta \mapsto \Theta^{\prime \prime}$ to the summands in (4.2) there remains no term if the sum is made over the ordered partitions of $[2 n]$. This proves identity (3.7). When making the sum over ordered partitions of $[2 n+1]$, the remaining terms correspond to the ordered partitions $\left(A_{0}, A_{1}, \ldots, A_{r}\right)$ having no increase and such that $A_{0}$ is a singleton. In particular, $r=n$. Such a partition is of the form $\left(\left\{a_{0}\right\},\left\{a_{1}<b_{1}\right\},\left\{a_{2}<b_{2}\right\}, \ldots,\left\{a_{n}<b_{n}\right\}\right)$ having the property 
that $a_{0}>a_{1}$ and for each $i=2, \ldots, n$ the relation $b_{i-1}>a_{i}$ holds. Hence $\omega=$ $a_{0} a_{1} b_{1} a_{2} b_{2} \cdots a_{n} b_{n}$ is a falling alternating permutation. We have then proved

$$
\sum_{\sigma \in \mathfrak{S}_{2 n+1}}(-1)^{n+\operatorname{lec} \sigma} q^{(\mathrm{inv}-\mathrm{lec}) \sigma}=\sum_{\omega \in \mathfrak{T}_{2 n+1}^{\prime}} q^{\text {inv } \omega} \quad(n \geq 0) .
$$

This establishes (3.8) by Theorem 5 .

\section{REFERENCES}

1. Désiré André, Développement de $\sec x$ et $\operatorname{tg} x$, C. R. Acad. Sci. Paris 88 (1879), 965-967.

2. Désiré André, Sur les permutations alternées, J. Math. Pures et Appl. 7 (1881), 167-184.

3. George E. Andrews and Dominique Foata, Congruences for the q-secant number, Europ. J. Combin. 1 (1980), 283-287. MR.595926 (82d:05018)

4. George E. Andrews and Ira Gessel, Divisibility properties of the q-tangent numbers, Proc. Amer. Math. Soc. 68 (1978), 380-384. MR0462960 (57:2925)

5. Leonard Carlitz, q-Bernoulli and Eulerian numbers, Trans. Amer. Math. Soc. 76 (1954), 332-350. MR0060538 (15:686a)

6. Leonard Carlitz, A combinatorial property of q-Eulerian numbers, Amer. Math. Monthly 82 (1975), 51-54. MR0366683 (51:2930)

7. Jacques Désarménien, Une autre interprétation du nombre de dérangements, Sémin. Lothar. Combin. 229/S-08 (1984), 11-16. (http://igd.univ-lyon1.fr/ slc/)

8. Leonhard Euler, Institutiones calculi differentialis cum eius usu in analysi finitorum ac Doctrina serierum, Academiae Imperialis Scientiarum Petropolitanae, St. Petersbourg, chap. VII (Methodus summandi superior ulterius promota), 1755.

9. Dominique Foata, Further divisibility properties of the q-tangent numbers, Proc. Amer. Math. Soc. 81 (1981), 143-148. MR.589157 (81k:05005)

10. Dominique Foata and Guo-Niu Han, Fix-Mahonian calculus. III. A quadruple distribution, Monatsh. Math. 154 (2008), 177-197. MR2413301 (2009b:05017)

11. Dominique Foata and Marcel-Paul Schützenberger, Théorie géométrique des polynômes eulériens, Lecture Notes in Math., 138, Springer-Verlag, Berlin, 1970. MR0272642 (42:7523)

12. George Gasper and Mizan Rahman, Basic hypergeometric series, Encyclopedia of Math. and its Appl., 35, Cambridge Univ. Press, Cambridge, 1990. MR.1052153 (91d:33034)

13. Ira Gessel, A coloring problem, Amer. Math. Monthly 98 (1991), 530-533. MR.1109577 (92h:05053)

14. J. H. Jackson, A basic-sine and cosine with symbolic solutions of certain differential equations, Proc. Edinburgh Math. Soc. 22 (1904), 28-39.

15. Percy Alexander MacMahon, Combinatory Analysis, vols. 1 and 2, Cambridge Univ. Press, Cambridge, 1915, 1916. (Reprinted by Chelsea, New York, 1960.) MR0141605 (25:5003)

16. Niels Nielsen, Traité élémentaire des nombres de Bernoulli, Gauthier-Villars, Paris, 1923.

17. John Riordan, An Introduction to Combinatorial Analysis, John Wiley, New York, 1958. MR0096594 (20:3077)

18. David Roselle, Permutations by number of rises and successions, Proc. Amer. Math. Soc. 19 (1968), 8-16. MR.0218256 (36:1343)

19. John Shareshian and Michelle L. Wachs, q-Eulerian polynomials: excedance number and major index, Electron. Res. Announc. Amer. Math. Soc. 13 (2007), 33-45. MR2300004 (2008e:05010)

20. Richard P. Stanley, Binomial posets, Möbius inversion, and permutation enumeration, J. Combinatorial Theory Ser. A 20 (1976), 336-356. MR.0409206 (53:12968)

Institut Lothaire, 1 Rue Murner, F-67000 Strasbourg, France

E-mail address: foata@math.u-strasbg.fr

Institut de Recherche Mathématique Avancée, UMR 7501, Université de Strasbourg et CNRS, 7 rue René-Descartes, F-67084 Strasbourg, France

E-mail address: guoniu@math.u-strasbg.fr 